\title{
Aproximación Metodológica para la Evaluación del Riesgo de Salinización de Suelos en Zonas Bajo Riesgo
}

\author{
Methodological approach for the Assessment \\ of Risk of Salinization of Soil in Low Irrigation Zones
}

\author{
Andrés Echeverri-Sánchez \\ Candidato PhD Ingeniería, Universidad del valle \\ andres.echeverri@correounivalle.edu.co \\ Cristo Facundo Pérez \\ Joven Investigador, Universidad del Valle \\ crifep@gmail.com \\ Norberto Urrutia-Cobo \\ Profesor Titular, Universidad del Valle \\ norberto.urrutia@correounivalle.edu.co
}

\section{Resumen}

La gestión del riesgo es el conjunto de elementos, medidas y herramientas dirigidas a la intervención de la amenaza o vulnerabilidad, con el fin de disminuir o mitigar tanto los riesgos existentes de desastres naturales y socio-naturales, así como disponer las estrategias para su manejo y recuperación. En Colombia la gestión del riesgo ha sido orientada totalmente fenómenos amenazantes como sismos, erupciones volcánicas e inundaciones. Solo en pocos casos se han abordado peligros de inicio lento como sequía. En el caso de estudio, se plantea una metodología para abordar el riesgo de degradación química de suelos en uno de los distritos de riegos más importantes de Colombia. De esta manera, se presenta la aproximación conceptual y metodológica desarrollada para evaluar el suelo como elemento expuesto ante una amenaza específica. Se propone evaluar y zonificar la amenaza mediante la caracterización de la calidad del agua de riego. Por otro lado, la evaluación de la vulnerabilidad integra aspectos intrínsecos del suelo (aspectos de fragilidad) y condiciones extrínsecas (aspectos de exposición). Finalmente evalúa el riego como el producto de la amenaza y la vulnerabilidad.

Palabras clave: agricultura de irrigación, degradación de suelos; gestión del riesgo; salinidad.

\section{Abstract}

Risk management is the set of elements, measures, and tools addressed to the intervention of threat or vulnerability, with the purpose of reducing or mitigating both existing risks of natural and socionatural disasters, as well as disposing the strategies for its management and recovery. Risk management in Colombia has been totally oriented to threatening phenomena such as earthquakes, volcanic eruptions and floods. Only in a few cases have the dangers of slow beginning such as droughts been addressed. In the case study, a methodology to address risk of chemical degradation of soils in one of the most important irrigation districts of Colombia is stated. This way, a conceptual and methodological approach is presented, developed to assess soil as an element exposed in a specific threat. Assessment and zoning of the threat is proposed through the characterization of the quality of water for irrigation. On the other side, assessment of vulnerability integrates intrinsic aspects of the soil (fragility aspects) and extrinsic aspects (Exposure aspects). Finally, it assesses irrigation as the product of threat and vulnerability.

Key Words: irrigation agriculture, degradation of soils; risk management; salinity.

\section{Introduction}

La producción de alimentos y el uso del agua están íntimamente relacionados. El agua siempre ha sido el principal factor limitante de la producción agrícola en gran parte del mundo donde la lluvia es insuficiente para satisfacer las necesidades de los cultivos. Con la creciente competencia por las fuentes hídricas disponibles y con el constante aumento de la demanda de productos básicos agrícolas, nunca antes había sido más urgente el llamado a mejorar la eficiencia y productividad del uso del agua en la agricultura, para garantizar con ello la seguridad alimentaria futura y hacerle frente a las incertidumbres asociadas al cambio climático (Steduto et al., FAO, 2012). 
Actualmente la agricultura consume alrededor de dos terceras partes del agua dulce en todo el mundo (FAO, 2013). El riego ha sido crucial para aumentar la producción de alimentos. El 20\% del área cultivada bajo agricultura de regadío, produce el $40 \%$ de la producción mundial de alimentos (FAO, 2007; FAO, 1996 citado por Corcoran et al., 2010). Adicionalmente, el riego reduce el riesgo a la sequía y fomenta la diversificación de cultivos, mejorando de ésta manera los ingresos rurales. Sin embargo, la mala administración del riego, también puede contribuir al anegamiento y salinización. El anegamiento del suelo proviene de la sobre-irrigación e inadecuado drenaje. De esta manera, se restringe el crecimiento de la planta y a menudo precede a la salinización. En general, la salinización resulta de la acumulación de sólidos disueltos en el suelo, y también pueden ocurrir en zonas encharcadas con suelos inherentemente susceptibles. El cambio climático hace que la solución de estos problemas sea más urgente. Las medidas apropiadas pueden ser implementadas para limitar la extracción excesiva, el anegamiento, y la salinización que pueda dar lugar a pérdidas considerables de tierras con vocación agrícola y resultar en costos operativos insostenibles (FAO, 2013).

El aumento de la concentración de sales en la solución del suelo, disminuye la disponibilidad del agua para la plantas a causa del aumento del potencial osmótico. Afecta de manera irreversible los procesos fisiológicos, impactando negativamente en la producción agrícola. Este tipo de degradación, es un proceso lento pero latente, que cada vez crece. Sin embargo, hasta el momento no se le ha prestado ningún tipo de atención ya que no existen planes de contingencia diseñados para mitigar su impacto. De hecho, una herramienta tan útil como la gestión del riesgo, ha sido totalmente enfocada a elementos expuestos como vidas o infraestructuras, y no se ha considerado el recurso suelo como un elemento expuesto; ignorando su importancia en el ámbito económico, social y ambiental. En este sentido, mientras el proceso avanza, el efecto recae sobre los ecosistemas, la productividad y sobre la economía de los productores agrícolas.

Teniendo en cuenta lo anterior, ni en Colombia, ni el Valle del Cauca ni en el distrito de riego RUT, existe un estimativo actual del área afectada por la salinidad, ni mucho menos uno que indique cuales zonas son más vulnerables a sufrir por ésta. Esto se debe a que no se dispone de suficiente información, la poca con la que se cuenta no está actualizada, y no existe una metodología que permita estimar la vulnerabilidad del suelo ante el potencial peligro de un agente externo, como pudiera ser el agua de riego. Bajo estas premisas, éste trabajo pretende socializar los avances conceptuales y metodológicos logrados hasta el momento por el grupo de trabajo, tendientes a conocer en profundidad el riesgo de salinización de suelos como herramienta para la toma de decisiones. Inicialmente se presentará un marco conceptual sobre las temáticas asociadas a la problemática asociada. Posteriormente se desarrollará la aproximación metodológica para la evaluación y zonificación de la amenaza, la vulnerabilidad y el riesgo de salinización de suelos en la zona de estudio.

\section{Marco Conceptual}

\section{Degradación del Suelos}

La degradación del suelo afecta su capacidad productiva y el medio ambiente, impactando de manera importante sobre la seguridad alimentaria y sobre la calidad de vida de los seres humanos. Según (Eswarán et al., 2001), la degradación de un suelo, puede ser enmascarada hasta cierto punto con el uso de productos y algunas prácticas, pero en algún momento los impactos negativos salen a flote. Esta falsa seguridad y la lentitud de los procesos de degradación, no ha permitido que el mundo se preocupe por un plan internacional que se ocupe de prevenir y mitigar la degradación de los suelos. De hecho, la evaluación de la degradación de suelos agrícolas y tierras es un tema que recientemente se viene discutiendo por los diferentes organismos internacionales (FAO, 1997), inclusive en la reciente entrega del Grupo Intergubernamental de Expertos sobre el Cambio Climático (IPCC, 2012) no se abordó la existente relación entre cambio climático y degradación de suelos. A nivel mundial los principales proceso de degradación del suelo son: erosión hídrica, compactación, salinización y reducción de la fertilidad. Estos procesos incluyen acciones físicas, químicas y biológicas que afectan la capacidad del suelo para su autorregulación y productividad (Lal et al., 1989 citado por de Paz et al., 2006).

\section{Salinidad en Suelos}

Dentro de los suelos salinos, se distinguen dos situaciones particulares según el catión predominante en el complejo de cambio: $\mathrm{Ca}^{2+} \mathrm{O} \mathrm{Na}^{+}$. Cuando el catión predominante es el $\mathrm{Ca}^{2+}$, las sales solubles abundan en el suelo, esto aumenta la presión osmótica de la solución del suelo, hace difícil la extracción del agua por parte de la planta, genera estrés hídrico, y por tanto disminuye la productividad del cultivo. Estos suelos se les denominan salinos. Cuando el $\mathrm{Na}^{+}$es el catión dominante se produce la dispersión de las arcillas, lo que lleva a una destrucción de la estructura, toxicidad debida al sodio y/o bicarbonatos, elevación del pH por acciones de los carbonatos sódicos. Estos suelos son calificados como sódicos o alcalinos (IDEAM, 2002).

De acuerdo con el IDEAM (2000) citado por IDEAM (2002), el país tiene cerca de $87.000 \mathrm{~km}^{2}(7.7 \%)$ de sus tierras en diferentes categorías de intensidad de salinización. De las 299.226.6 ha de la zona plana del valle geográfico del río Cauca dedicadas a la agricultura, 85.000 (28.4\%) se encontraban afectadas en los años ochenta por salinidad y mal drenaje (IGAC, IDEAM, MAVDT, 2010). En el distrito de riego RUT existe algún problema de salinidad en al menos 3600 hectáreas (36\% del área) en el primer metro de profundidad (Romero, 2013). 


\section{Gestión del Riesgo}

La gestión del riesgo es el conjunto de elementos, medidas y herramientas dirigidas a la intervención de la amenaza o la vulnerabilidad, con la finalidad de disminuir o mitigar los riesgos existentes (Cardona, 2005). Sin embargo, el marco conceptual asociado a la interpretación del riesgo, no solo ha variado con el tiempo, también ha variado desde la perspectiva disciplinar desde la cual se ha abordado su noción (Cardona, 2003). Esta falta de consenso ha generado confusión entre investigadores y especialistas de las diferentes disciplinas, lo que ha impedido consolidar una terminología consistente. De acuerdo con Maskrey (1998), los modelos de riesgo de las ciencias naturales han sido básicamente modelos de amenazas. Mientras que en las ciencias sociales y/o medicina, el término de vulnerabilidad ha sido empleado para referirse directamente al riesgo (Maskrey, 1998; Cardona, 2003). Por el contrario, las ciencias aplicadas han desarrollado una perspectiva en la cual el riesgo de un elemento expuesto ( $\mathrm{R}_{\mathrm{e}}$ ) está en función de la amenaza (A) y la vulnerabilidad (V) (Ec. 1) (Maskrey, 1998; Cardona, 1993).

$$
R_{e}=f(A, V)
$$

De acuerdo con esto último, se acepta que el concepto de amenaza se refiere a un peligro latente o factor de riesgo externo de un sistema o de un sujeto expuesto. Mientras, la vulnerabilidad se entiende, como un factor de riesgo interno del elemento expuesto a una amenaza, por lo que corresponde a la predisposición de ser afectado o susceptibilidad de sufrir daños por el fenómeno que caracteriza la amenaza. De esta manera, el riesgo corresponde al potencial de perdida que pueden ocurrirle al sujeto o sistema expuesto, resultado de la convolucion de la amenaza y la vulnerabilidad (Cardona, 2003). En este sentido, algunos autores plantean que conceptualmente la evaluación del riesgo (Re) puede ser expresado como el producto de la amenaza por la vulnerabilidad (Wilches-Chaux, 1998; Maskrey, 1998; UNISDR, 2009) (Ec. 2).

$$
R_{e}=A^{*} V
$$

La nulidad de uno de los dos factores hace nulo el producto (el riesgo). Si no hay una amenaza determinada, no habrá riesgo aunque el elemento expuesto sea vulnerable a esa amenaza. De igual modo, si no existe vulnerabilidad, no existirá el riesgo ante una amenaza específica. Por lo que ninguno de esos dos factores, aisladamente, podría dar lugar ni al riesgo ni al desastre. Sin embargo es importante tener en cuenta que la realidad es mucho más compleja que una mera multiplicación aritmética, al igual que un verdadero sancocho es mucho más complejo que el resultado de simplemente sumar yuca + papa + mazorca + gallina + agua. (Wilches-Chaux, 1998).

De acuerdo con lo anterior, algunos autores analizan el riesgo partiendo de la ecuación 2 , pero la evaluación del riesgo la realizan de una forma diferente.
Taubenböck et al., (2008), afirman que la ecuación 2 representa la dependencia de ambos componentes (A, V), pero la evaluación del riesgo lo hacen a través de una adición $[\mathrm{Re}=1 / 2(\mathrm{~A}+\mathrm{V})]$ con la finalidad de obtener resultados entre 0-1 en vez de minimizar los valores usando una multiplicación.

\section{Evaluación de la Amenaza}

Evaluar la amenaza es "pronosticar" la ocurrencia del fenómeno con base en el estudio de su mecanismo generador, el monitoreo del sistema perturbador $y / 0$ el registro de eventos en el tiempo. Un pronóstico puede ser a corto, mediano y largo plazo. Así mismo, en mapas de diferentes escalas se plasma la cuantificación de la amenaza y se lleva a cabo una "zonificación" en la cual, mediante un proceso de determinación de la misma en varios sitios, delimitan áreas homogéneas o zonas de amenaza constante. A este tipo de cartografía se le conoce como mapas de amenaza, los cuales son un insumo de importancia para la planificación física y territorial (Cardona, 1993).

\section{Análisis y Evaluación de la Vulnerabilidad}

Cardona (2006), ha propuesto un modelo para evaluar el riesgo de desastres desde una perspectiva holística. Desde esta perspectiva es importante considerar valorar la vulnerabilidad bajo tres factores o categorías. i) el grado de exposición y/o susceptibilidad física, dependiente de la amenaza; ii) la fragilidad, independiente de la amenaza pero relacionada con el impacto potencial sobre el contexto del elemento expuesto; y iii) la resiliencia, que se refiere al nivel de asimilación o la capacidad de recuperación ante una amenaza. En este sentido, para capturar toda su complejidad, es importante evaluar la vulnerabilidad desde enfoques tanto cualitativos como cuantitativos ya que hay aspectos de la vulnerabilidad que son dependientes de la amenaza y otros que no lo son pero que agravan la situación (Cardona, 2006; Birkmann, 2007; Cardona, 2012). Es substancial reconocer que los sistemas complejos envuelven múltiples variables (física, social, cultural, económica y ambiental) que no pueden ser medidos usando la misma metodología. Esto indica que la ponderación y medición del riesgo envuelve la integración de diversas perspectivas disciplinarias (de Lange et al., 2010; Cardona et al., 2012).

De acuerdo con lo anterior, uno de los principales problemas en la gestión del riesgo, no es el riesgo en sí, sino poder identificar y evaluar los factores asociados a la vulnerabilidad del elemento expuesto. En muchas ocasiones los componentes de esta vulnerabilidad son variables tanto cualitativas como cualitativas. En este sentido, se propone una metodología que podría ser muy útil para la toma de decisiones en problemas agroambientales. El Análisis Multi-criterio (Saaty, 1987; 1990), en el cual, además de valorar la importancia relativa de un parámetro sobre otro, se obtienen los grados de importancia de cada parámetro, se integran al análisis aquellos que son cualitativos y se tiene en cuenta la opinión de expertos en las temáticas 
asociadas al análisis del problema (Saaty, 2008). Esto se combinará con un SIG (análisis geoestadístico), herramienta más idónea empleada para el manejo, manipulación, procesamiento, mapeo y especialmente organización de datos con el fin de facilitar el análisis en la vulnerabilidad o degradación de suelos (de Paz et al., 2006; Juan et al., 2011; Salvati et al., 2011; Zabeo et al., 2011).

\section{Aproximación Metodológica Propuesta}

\section{Evaluación y Zonificación de la Amenaza}

En las zonas irrigadas, el factor amenazante está representado en el agua de riego, por tanto la evaluación de la amenaza de salinización se debe fundamentar en el estudio de la calidad del agua. La conductividad eléctrica ha sido usada como indicador universal de la concentración salina del agua de riego (Ayers and Westcot, 1985). Por otro lado, se conoce que las sales más solubles representan menor peligro que las sales menos solubles (Pizarro, 1996).

Teniendo en cuenta lo anterior y otras particularidades de las zonas bajo riego en Colombia, se propone el siguiente procedimiento general para la valoración del peligro o amenaza de salinización de suelos.

- Zonificación del área bajo riego en función de la fuente hídrica: Si el distrito no tiene una única fuente hídrica, debe zonificarse en función de las distintas fuentes. La red hidráulica será de gran ayuda para esta zonificación.

- Diseño de muestreo de calidad de agua: El muestreo debe diseñarse de tal manera que la toma de muestras se lleve a cabo el mismo día, durante las horas en las que se lleva a cabo la actividad del riego. En cada sitio deben tomarse como mínimo 3 muestras y si se cuenta con recursos suficientes, tomar 3 muestras dos o tres veces el mismo día. Se debe realizar un muestreo para cada época representativa de precipitación, es decir, como mínimo debe muestrearse en una época de lluvias y una de estiaje. Para cada zona identificada se deben seleccionar como mínimo 3 puntos representativos. Los parámetros a muestrear son los siguientes:
$\mathrm{CEw}, \mathrm{pH}, \mathrm{Ca}, \mathrm{Mg}, \mathrm{K}, \mathrm{Na}, \mathrm{SO}_{4}, \mathrm{HCO}_{3}, \mathrm{Cl}$.

- Muestreo y Procesamiento de laboratorio: El muestreo debe realizarse siguiendo los protocolos presentados por APHA (1999).

- Calificación de peligro: La calificación de peligro de amenaza debe realizarse mediante una matriz de decisión en la que se tenga en cuenta el valor de CE del agua y la composición salina dominante. Para el primer caso se sugiere seguir los lineamientos de FAO (Ayers and Westcot, 1985) y en el segundo caso, utilizar un modelo predictivo de composición salina de agua de riego, tal como SOSALRIEGO (Villafañe, 2011). Una propuesta de esta matriz es presentada en la Tabla 1.

Tabla 1. Matriz de decisión para calificar peligro de salinización

\begin{tabular}{|c|c|cc|}
\hline \multirow{2}{*}{ CEw dS $\mathbf{~ m}^{-1}$} & \multicolumn{3}{|c|}{ Solubilidad de sales en agua $\left(\mathbf{m m o l}_{\mathbf{c}} \mathbf{~}^{\mathbf{1}}\right)$} \\
\cline { 2 - 4 } & $>\mathbf{5 0 0 0}$ & $\mathbf{1 0 0 0 - 5 0 0 0}$ & $<\mathbf{1 0 0 0}$ \\
\hline $\mathbf{0 - 0 . 2 5}$ & $\mathrm{L}$ & $\mathrm{L}$ & $\mathrm{VL}$ \\
$\mathbf{0 . 2 5 - 0 . 7}$ & $\mathrm{M}$ & $\mathrm{M}$ & $\mathrm{L}$ \\
$\mathbf{0 . 7}-\mathbf{3 . 0}$ & $\mathrm{H}$ & $\mathrm{H}$ & $\mathrm{M}$ \\
$>\mathbf{3 . 0}$ & $\mathrm{VH}$ & $\mathrm{VH}$ & $\mathrm{H}$ \\
\hline
\end{tabular}

- Mapeo de resultados: El mapeo de resultados se realizará de acuerdo al número de zonas de calidad de agua identificadas. Mediante el uso de un software de manejo de cartografía digital es posible construir polígonos que representen a cada zona identificada y asignar la calificación de amenaza mediante la edición de la tabla de atributos de la capa. De esta manera será posible visualizar la distribución espacial de la amenaza y su variación temporal.

\section{Evaluación y Zonificación de la Vulnerabilidad}

- Conceptualización del problema e identificación de parámetros: De acuerdo a la literatura, los parámetros más mencionados como asociados a la vulnerabilidad del suelo a los procesos de salinización se tienen los siguientes (Tabla 2).

Tabla 2. Componentes de la Vulnerabilidad

\begin{tabular}{|c|c|c|c|}
\hline Tipo & & Factor & Indicador \\
\hline \multirow{5}{*}{ 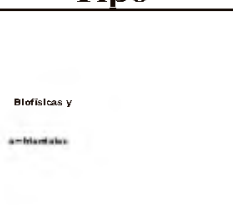 } & \multirow{2}{*}{ Fragilidad } & 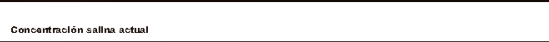 & CEw \\
\hline & & Textura del suelo & $\%$ de arcillas, limos y arenas \\
\hline & \multirow{3}{*}{ Exposición } & Aporte de sales freáticas & Profundidad nivel freático \\
\hline & & Pendiente del Terreno & $\%$ de pendiente \\
\hline & & Relación Precipitación/ETo & Índice de aridez \\
\hline \multirow{4}{*}{$\begin{array}{c}\text { Socio- } \\
\text { Económicas }\end{array}$} & \multirow{4}{*}{ Exposición } & Prácticas de fertilización & Cualitativo \\
\hline & & Eficiencia de aplicación del agua & Ea \\
\hline & & Patrón de distribución del agua & Cualitativo \\
\hline & & Infraestructura de drenaje & Cualitativo \\
\hline
\end{tabular}


- Asignación de grados de importancia a parámetros identificados: Se realiza mediante la consulta de expertos, en la que se realiza una calificación parada de los parámetros identificados en la etapa anterior. Se utiliza la escala de valores de Saaty (2008), en la que el valor 1 significa que el parámetro evaluado tiene igual importancia que el parámetro con el cual se compara. El valor 9 significa que el parámetro evaluado es absolutamente más importante que el parámetro de comparación. Una vez realizada la calificación de todos los expertos, es posible obtener los pesos o grados de importancia de cada parámetro considerado y al mismo tiempo un valor representativo de la consistencia de la calificación del grupo de expertos (Índice de Consistencia IC). Valores de IC menores a 10 son aceptables (Manyoma, 2015).

- Categorización y normalización de parámetros: Los parámetros identificados deben dividirse en categorías en función del potencial impacto sobre el problema analizado. La literatura será de gran ayuda en este aspecto. Seguidamente debe realizarse el proceso de normalización, de tal manera que tanto para los parámetros cuantitativos como para los cualitativos, la categoría con mayor impacto sobre la vulnerabilidad del suelo a la salinización debe tener una valoración de 1. Las demás categorías obtendrán valores menores a 1.

- Generación de índice de vulnerabilidad a la salinización de suelos: Una vez normalizados todos los parámetros, se procede a obtener planos en formato raster de cada uno de ellos. Este proceso se realiza en un software de geo procesamiento espacial como por ejemplo el ArcGis. Debe definirse una misma área de procesamiento y un mismo tamaño de celda o pixel para todos los parámetros. La aplicación del modelo se realiza para cada celda mediante el uso de la siguiente expresión:

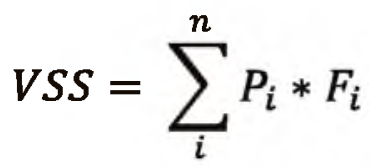

Donde,

IVSS : índice de vulnerabilidad del suelo a procesos de salinización

Pi : Peso promedio asignado al parámetro i.

Fi : Valor normalizado del parámetro i en la celda analizada.

La etapa final del proceso contempla la reclasificación de los valores obtenidos para el IVSS y su agrupamiento en categorías de vulnerabilidad Muy Baja, Baja, Media, Alta y Muy Alta.

\section{Evaluación y Zonificación del Riesgo}

Para esta fase se aplicará el concepto de algebra de mapas en formato "raster", donde la evaluación del riesgo es igual al producto de los mapas de Amenazas por el de la Vulnerabilidad (Figura 1). Las categorías de amenaza recibirán calificaciones de 1 hasta 5 , siendo 1 "Muy Baja" y 5 "Muy Alta". De igual manera se asignarán valores a las categorías de vulnerabilidad. Lo anterior permitirá identificar claramente los resultados obtenidos del proceso de multiplicación de las superficies raster de amenaza y vulnerabilidad. Se tendrán valores entre $2(1 \times 1)$ y $25 \quad(5 \times 5)$. La reclasificación del nivel de riesgo de salinización de suelos puede realizarse de la siguiente manera:

Riesgo Bajo: Valores menores a 10

Riesgo Medio: Valores entre 10 y 18

Riesgo Alto: Valores mayores a 18

Figura 1. Evaluación del Riesgo
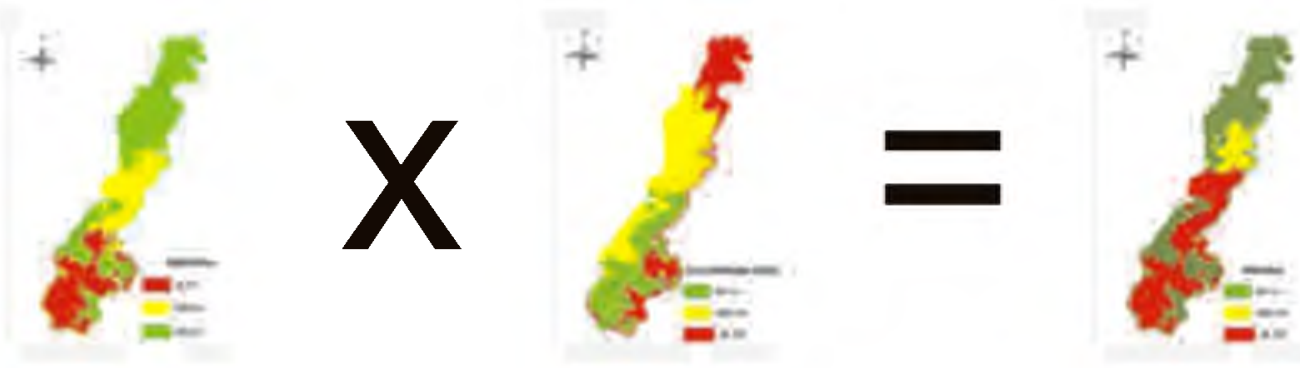

\section{Consideraciones Finales}

De acuerdo a los conceptos revisados y la aproximación metodológica expuesta se concluye que es posible utilizar esta herramienta en la gestión del riesgo de degradación de suelos en general y su replicación en otras zonas del país con otros tipos de amenaza y degradación.

La calidad de los resultados a obtener estará ligada a la calidad de la información de entrada en la metodología (se usará información secundaria y primara). Busca integrar información que posiblemente exista en diferentes fuentes (instituciones estatales y privadas, organizaciones comunitarias y unidades académicas).

La metodología considera aspectos técnicos, ambientales y culturales en el análisis de la vulnerabilidad, pero 
adolece de económicos, social y organizacional, considerados por varios autores.

\section{Agradecimientos}

Los autores expresan su agradecimiento a COLCIENCIAS por la financiación de Andrés Echeverri y Cristo Pérez como becarios de las bolsas Doctorado Nacional y Joven Investigador, respectivamente. A la Universidad del Valle, el Grupo REGAR - Escuela EIDENAR, ASORUT y CVC por facilitar la realización de este estudio.

\section{Referencias Bibliográficas}

APHA. (1999). Standard Methods for the Examination of Water and Wastewater. (L. S. Clesceri, A. E. Greenberg, \& A. D. Eaton, Eds.) American Public Health, Association American Water Works Association, Water Environment Federation (20th ed., Vol. 20th). Washington, DC: Amer Public Health Assn.

Ayers, R.; Westcot, D. (1985).Water Quality for Agriculture. Irrigation and Drainage Technical Paper No 29. FAO. Rome, Italy.

Birkmann, J., (2007). Risk and vulnerability indicators at different scales: Applicability, usefulness and policy implications. Environmental Hazards 7, 20-31.

Cardona, Omar. (1993). Evaluación de la Amenaza, la Vulnerabilidad y el Riesgo "Elementos para el Ordenamiento y la Planeación del Desarrollo". En: Los desastres No Son Naturales. Andrew Maskrey (editor). Ediciones tercer mundo. Bogotá. Páginas 45-65.

Cardona, Omar. (2003). The need for rethinking the concepts of vulnerability and risk from a Holistic Perspective: A necessary review and criticism for effective risk management. Chapter 3 of the book "Mapping Vulnerability: Disasters, Development and People". G. Bankoff, G. Frerks, D. Hilhorst (Editors). Earth scan Publishers, London.

Cardona, Omar. (2005). La gestión del Riesgo Colectivo: Un marco conceptual que encuentra sustento en una ciudad laboratorio. Evento: Seminario Internacional Involucrado a la Comunidad en los Programas de Reducción de Riesgos, UDEFA, Punto Fijo, Estado de Falcón, Venezuela. [en línea]. En: http://www.desenredando.org/public/articulos/2007/articulos _omar/Gestion_Riesgo_Ciudad_Laboratorio21-09-05LaRED.pdf. Citado el 13 de septiembre de 2013.

Cardona, Omar. (2006). "Midiendo lo Inmedible" Indicadores de Vulnerabilidad y Riesgo. Boletín Ambiental 53. Instituto de Estudios Ambientales IDEA, Universidad Nacional de Colombia, sede Manizales. Manizales, Colombia.

Cardona, Omar D., M.K. van Aalst, J. Birkmann, M. Fordham, G. McGregor, R. Perez, R.S. Pulwarty, E.L.F. Schipper, and B.T. Sinh. (2012). Determinants of risk: exposure and vulnerability. In Managing the Risks of Extreme Events and Disasters to Advance Climate Change Adaptation [Field, C.B., V. Barros, T.F. Stocker, D. Oin, D.J. Dokken, K.L. Ebi, M.D. Mastrandrea, K.J. Mach, G.-K Plattner, S.K. Allen, M. Tignor, and P.M. Midgley (eds.)]. A Special Report of Working Groups I and II of the Intergovernmental Pane on Climate Change (IPCC). Cambridge University Press, Cambridge, UK, and New York, NY, USA, pp. 65-108.

Corcoran, E., Nellemann, C., Baker, E., Bos, R., 2010. Sick water? The central role of wastewater management in sustainable development. A rapid response assessment. Water.

Eswaran, H., R. Lal and P.F. Reich. (2001). Land degradation: an overview. In: Bridges, E.M., I.D. Hannam, L.R. Oldeman, F.W.T Pening de Vries, S.J. Scherr, and S. Sompatpanit (eds.). Responses to Land Degradation.Proc.2nd.International Conference on L.and Degradation and Desertification, KhonKaen, Thailand.Oxford Press, New Delhi, India.

Food and Agriculture Organization of the United Nations - FAO. (1997) Land quality indicators and their use in sustainable agriculture and rural development. Land and Water Bulletin No. 5. FAO, Rome, $212 \mathrm{pp}$.

FAO. (2007).Water at a glance: The relationship between water agriculture, food security and poverty. [en línea]. En: http://www.fao.org/nr/water/docs/waterataglance.pdf. Citado el 13 de septiembre de 2013.

FAO. (2013). FAO Statistical Yearbook: World Food and Agriculture. [en líneal. En:

http://www.fao.org/docrep/018/i3107e/i3107e00.htm. Citado el 13 de septiembre de 2013.
GTZ. (2006). Conceptos Asociados a la Gestión del Riesgo de Desastre en la Planificación e Inversión para el Desarrollo. Programa Desarrollo Rural Sostenible de la Cooperación Técnica Alemana GTZ. 64 p.

IDEAM. (2002). Zonificación de los Procesos de Salinización de los Suelos de Colombia. Subdirección de Geomorfología y Suelos. $43 p$ p. Bogotá, Colombia.

IGAC; IDEAM; MAVDT. (2010). Protocolo para la Identificación y Evaluación de los Procesos de Degradación de Suelos y Tierras por Salinización. Bogotá D.C. 323 pp.

IPCC. (2012). Managing the Risks of Fxtreme Events and Disasters to Advance Climate Change Adaptation. A Special Report of Working Groups I and II of the Intergovernmental Panel on Climate Change [Field, C.B., V. Barros, T.F. Stocker, D. Oin, D.J. Dokken, K.L. Ebi, M.D. Mastrandrea, K.J. Mach, G.-K. Plattner, S.K. Allen, M. Tignor, and P.M. Midgley (eds.)]. Cambridge University Press, Cambridge, UK, and New York, NY, USA, $582 \mathrm{pp}$.

de Lange, H.J., Sala, S., Vighi, M., Faber, J.H., (2010). Ecological Vulnerability in Risk Assessment- a Review and Perspectives. The Science of the total environment 408, 3871-9.

Juan, P., Mateu, J., Jordan, M.M., Mataix-Solera, J., Meléndez-Pastor, I., Navarro-Pedreño, J., (2011). Geostatistical Methods to Identify and Map Spatial Variations of Soil Salinity.Journal of GeochemicalExploration 108,62-72.

Manyoma-Velásquez, P. (2015). Localización de Rellenos Sanitarios Regionales en Áreas Urbanas de Rápido Crecimiento. Tesis Doctoral, Universidad del valle. Cali. Colombia.

Meza, L; Corso, S; Sosa, S. (2010). Gestión del Riesgo de Sequía y otros Eventos Climáticos Extremos en Chile. Organización de Las Naciones Unidas para la Agricultura y la Alimentación - FAO. Santiago. $128 \mathrm{p}$.

Narváez, L; Lavell, A; Pérez Ortega, G. (2009). La Gestión del Riesgo de Desastres: un Enfoque Basado en Procesos. Proyecto Apoyo a la Prevención de Desastres en la Comunidad Andina - PREDECAN. San Isidro, Perú.

de Paz, J.-M., Sánchez, J., Visconti, F. (2006). Combined use of GIS and Environmental Indicators for Assessment of Chemical, Physical and Biological Soil Degradation in a Spanish Mediterranean Region. Journal of environmental management, (79), 150-62.

Romero L., Gustavo. (2013). Estado Actual de la Salinidad de Los Suelos en el Valle del Río Cauca. Pág. 92-135. Tecnicaña (Asociación Colombiana de Técnicos de la Caña de Azúcar de Colombia). Memorias Curso-Taller: Modelos, prevención y recuperación de suelos con problemas de sales. Cali, Colombia.

Saaty, R. W. (1987). The analytic hierarchy process-what it is and how it is used. Mathematical Modelling, $9(3-5), 161-176$. http://doi.org/10.1016/0270-0255(87)90473-8

Saaty, T. L. (1990). How to make a decision: The analytic hierarchy process. European Journal of Operational Research, 48(1), 9-26. http://doi.org/10.1016/0377-2217(90)90057-I

Saaty, T. L. (2008). Decision making with the analytic hierarchy process. International Journal of Services Sciences, 1(1), 83. http://doi.org/10.1504/IJSSCI.2008.017590

Salvati, L., Bajocco, S., Ceccarelli, T., Zitti, M., Perini, L.. (2011). Towards a process-based evaluation of land vulnerability to soil degradation in Italy.EcologicalIndicators 11, 1216-1227.

Soldano, Alvaro. (2009). Conceptos sobre riesgo. Comisión nacional de actividades espaciales (CONAE). Departamento de Sostenible de la Secretaría General de la Organización de los Estados Americanos. Síntesis temática Foro Virtual RIMD. Córdoba. $5 p$.

Steduto, P.; Hsiao, T. C.; Fereres, E.; Raes, D. 2012. Crop Yield Response to Water. Food and Agriculture Organization of the United Nations -FAO, Irrigation and Drainage Paper 66. Rome, Italy, pp. 500.

Taubenböck, H; Post, P; Roth, A; Zosseder, K;Strunz, G; S. Dech. (2008). A Conceptual Vulnerability and Risk Framework as Outline to Identify Capabilities of Remote Sensing. Nat. Hazards Earth Syst. Sci., 8, 409-420.

UNISDR. (2009). Terminology on Disaster Risk Reduction. International Strategy for Disaster Reduction. [en líneal. En: http://www.unisdr.org/files/7817_UNISDRTerminologyEnglish. pdf. Citado el 13 de septiembre de 2013.

Villafañe, R. (2011). Sosalriego: un procedimiento para diagnosticar los riesgos de sodificación y salinización del suelo con el agua de riego (nota técnica). Bioagro, 23(1), 57-64.

Wilches-Chaux, Gustavo. (1998). Auge, caída y levantada de Felipe Pinillo, mecánico y soldador o yo voy a correr el riesgo. Guía de la red para la gestión local del riesgo. Red de Estudios Sociales en Prevención de Desastres en América Latina.

Zabeo, a, Pizzol, L., Agostini, P., Critto, a, Giove, S., Marcomini, a. (2011). Regional Risk Assessment for Contaminated Sites Part 1: Vulnerability Assessment by Multicriteria Decision Analysis. Environment international 37, 1295-306. 